\title{
Low mid-upper arm circumference identifies children with a high risk of death who should be the priority target for treatment
}

André Briend ${ }^{1,2^{*}}$, José-Luis Alvarez ${ }^{3}$, Nathalie Avril ${ }^{4}$, Paluku Bahwere ${ }^{5,6}$, Jeanette Bailey ${ }^{7}$, James A. Berkley ${ }^{8}$, Paul Binns ${ }^{9}$, Nikki Blackwell ${ }^{10}$, Nancy Dale ${ }^{11}$, Hedwig Deconinck ${ }^{12}$, Pascale Delchevalerie ${ }^{13}$, Nicky Dent ${ }^{14}$, Maureen Gallagher ${ }^{15}$, Saul Guerrero ${ }^{16}$, Kerstin Hanson ${ }^{17}$, Marko Kerac ${ }^{18}$, Mark Manary ${ }^{19,20}$, Martha K. Mwangome ${ }^{21}$, Mark Myatt ${ }^{22}$, Kevin P. Q. Phelan ${ }^{23}$, Silke Pietzsch ${ }^{24}$, Núria Salse Ubach ${ }^{25}$, Susan Shepherd ${ }^{26}$, Saskia van der Kam² Antonio Vargas ${ }^{28}$ and Sophie Whitney ${ }^{29}$

\begin{abstract}
Background: Severe acute malnutrition (SAM) is currently defined by the WHO as either a low mid-upper arm circumference (i.e. MUAC $<115 \mathrm{~mm}$ ), a low weight-for-height z-score (i.e. WHZ <- 3), or bilateral pitting oedema. MUAC and WHZ do not always identify the same children as having SAM. This has generated broad debate, as illustrated by the recent article by Grellety \& Golden (BMC Nutr. 2016;2:10).

Discussion: Regional variations in the proportion of children selected by each index seem mostly related to differences in body shape, including stuntedness. However, the practical implications of these variations in relation to nutritional status and also to outcome are not clear. All studies that have examined the relationship between anthropometry and mortality in representative population samples in Africa and in Asia have consistently showed that MUAC is more sensitive at high specificity levels than WHZ for identifying children at high risk of death. Children identified as SAM cases by low MUAC gain both weight and MUAC in response to treatment. The widespread use of MUAC has brought enormous benefits in terms of the coverage and efficiency of programs. As a large high-risk group responding to treatment, children with low MUAC should be regarded as a public health priority independently of their WHZ.

Conclusion: While a better understanding of the mechanism behind the discrepancy between MUAC and WHZ is desirable, research in this area should not delay the implementation of programs aiming at effectively reducing malnutrition-related deaths by prioritising the detection and treatment of children with low MUAC.
\end{abstract}

Keywords: Severe acute malnutrition, Mid-upper arm circumference, Weight-for-height, Mortality, Public health priority

\footnotetext{
* Correspondence: andre.briend@gmail.com

${ }^{1}$ Tampere Centre for Child Health Research, University of Tampere and

Tampere University Hospital, Lääkärinkatu 1, 33014 Tampere, Finland

${ }^{2}$ Department of Nutrition, Exercise and Sports, Faculty of Science, University

of Copenhagen, Rolighedsvej 30, DK-1958 Frederiksberg, Denmark

Full list of author information is available at the end of the article
} 


\section{Background}

The paper recently published by Grellety and Golden [1] and the accompanying commentary [2] sets out to inform the current debate about the optimum method of selection of acutely malnourished children in need of treatment. While it provides interesting information from the authors' own cross-sectional data, its interpretation of published data diverts from the objective of the debate, which is eliminating malnutrition related deaths, and threatens the substantial gains that have been made through the use of mid-upper arm circumference (MUAC) in community-based nutrition programs.

The WHO defines severe acute malnutrition (SAM) as either a MUAC less than $115 \mathrm{~mm}$ (in children aged 6-59 months) or a weight-for-height $\mathrm{z}$-score (WHZ) less than -3 using the 2006 WHO child growth standards, or by the presence of bilateral pitting edema. Grellety and Golden show that MUAC and WHZ identify overlapping but not identical groups of children, as previously reported in the seven studies quoted and many other papers of this kind [3-5] (not an exhaustive list). This paper is based on a large set of survey datasets and shows either MUAC or WHZ identifying more malnourished children in different regions of the world. The authors are probably correct in ascribing these variations to differences in body shape, stunting prevalence, fat distribution and leg length. These factors overlap as, for example, stunting is associated with shorter leg length, which also affects body shape and probably results in WHZ variation. Beyond the role of leg length mentioned in the paper, the low prevalence of wasting defined by WHZ in Brazilian children can be explained by larger head, chest and abdominal circumferences in relation to height compared to children in the reference population [6]. There is also evidence that in some parts of the world, children may have more fat than expected and that fat distribution in the body varies. The term "thin fat" phenotype associated with a higher proportion of central fat and increased insulin resistance has, for example, been proposed to describe the situation in South Asia [7, 8].

Interpretation of these variations in body shape is difficult, however, as the mechanism underlying these differences in body proportion and their clinical implications in terms of outcome are not clear. For instance, "long legs", which increases the proportion of children with a low WHZ is found mainly in wealthier families and has been associated with good health [9]. It has been suggested that central fat (influencing WHZ) is predominantly involved in the regulation of the immune response and higher expression of cytokine genes, while peripheral fat (more influencing MUAC) seems more closely related to leptin which also plays a role in the regulation of the immune response but is also linked to survival $[10,11]$.

MUAC is being increasingly used in health and nutrition programs both in the community and at the health facility level because in addition to its simplicity of use it is very good at identifying children with a high risk of death if left untreated. All community studies that compared MUAC and WHZ to identify high-risk children in the absence of treatment have shown MUAC to be superior to WHZ. An extensive review published in 1994 on the relationship between anthropometry and mortality already noted that: "in comparing across studies and across the various comparative criteria, the most consistent observation is that WHZ is the least effective predictor of mortality. For the criterion that is common to all studies (ROC curves), it appears that at high specificities simple MUAC is superior to height-for-age and weight-for-age." [12]. These results were not changed by the introduction of the 2006 WHO child growth standards $[13,14]$. This has also been confirmed in children less than 6 months old at the time of vaccination who are in a very narrow age range suggesting the association with mortality is not entirely due to an age effect [15]. The relative utility of MUAC compared to WHZ has been demonstrated in settings as diverse as Bangladesh (in two separate studies) [16, 17] Uganda [18] Senegal [13], Democratic Republic of Congo [19] and the Gambia [15]. Thus, Golden and Grellety are incorrect in asserting that this association relies solely on data from Bangladesh and Malawi. The statement is based on an incomplete review of the literature - only a single community study comparing the association of MUAC and WHZ with mortality is quoted in their paper.

Against the results of all these highly consistent community studies, Grellety and Golden present two studies based solely on data from therapeutic treatment programs. Such studies are not adequate to make policy recommendations as their study populations do not reflect a general population; data are taken from a nutritional program that draws its participants from community-based screening, as is explicitly stated in one of these papers [20]. This is particularly a concern for comparisons of the association of WHZ and MUAC with mortality when screening is predominantly based on one of these criteria, biasing the sample.

The first study from South Sudan [21] was based on a truncated sample as only $0.9 \%$ of children with SAM were identified by MUAC alone. This is highly unrepresentative of the common situation - as in the just published paper under discussion the proportion of children with MUAC less than $115 \mathrm{~mm}$ (and WHZ >-3) among those with SAM was on average $39.3 \%( \pm 18.2)$ (see Table 3 in [1]). This almost complete absence of children selected only with MUAC in this sample was the result of a patient selection process whereby children 6 months of age or older but less than $65 \mathrm{~cm}$ in length were only eligible for admission if their WHZ was less than -3; their eligibility was not based on MUAC alone. This led 
to a drastically reduced proportion of high-risk low MUAC children. In this truncated sample, $37 \%$ of deaths occurred in children not identified by MUAC less $115 \mathrm{~mm}$. Grellety and Golden contrast this to the $98 \%$ of deaths in children identified by WHZ, overlooking the fact that in the initial sample a little above $99 \%$ of children had a WHZ less than -3. Even in this truncated sample from which the most high risk children with low MUAC were absent, children admitted by MUAC less than $115 \mathrm{~mm}$ had a higher risk of death compared to those admitted with a WHZ less than -3 and a MUAC greater than $115 \mathrm{~mm}(\mathrm{RR}=4.3 ; 95 \% \mathrm{CI}: 2.23-8.27)$ confirming that MUAC preferentially selects high risk children. This is consistent with the close association observed between MUAC and mortality previously observed in unselected hospital samples [22-24].

Grellety and Golden also claim that MUAC and WHZ are complementary and additive, rather than alternative measures that compete to identify the same individual children at increased risk of death. They support this claim with a second study from a treatment program which showed that "children with both a deficit in MUAC and WHZ have a worse prognosis than those with a single anthropometric deficit" [20]. This is, however, not supported by the data from the study they quote. Mortality was virtually the same in the two groups (4/929 vs. 6/940, $p=0.54$ for single and combined deficits respectively). The major difference between the two groups was a lower proportion of recovery in children with combined deficit compared to those with only low MUAC (71\% vs. $56 \%, p<0.01$ ). However, since WHZ was part of the definition of recovery, this is likely to be a circular argument as children with combined deficit started treatment with a lower WHZ and were more likely to need additional time to reach this WHZ based recovery criterion [25]. Nor does the higher weight gain observed in this study in children with combined deficit fit with the hypothesis of a poorer prognosis. The hypothesis of an independent effect of low MUAC and low WHZ on mortality is not consistent with either community follow-up of untreated children from Bangladesh and Senegal which showed that for a given MUAC, taller children (who have a lower WHZ) have a significantly better survival $[26,27]$.

In conclusion, this paper provides some interesting information on the geographical distribution of low MUAC compared to low WHZ. This finding clearly begs an explanation. We need more studies on differences in body shape, going beyond the simple measure of leg length. We need more studies comparing body composition, especially muscle mass and distribution of body fat and their relationship with outcome. Hopefully this paper by Grellety and Golden will stimulate research in this area. This paper does not however present any new information on the relationships between anthropometry and outcome, which is the basis of diagnostic criteria, and fails to highlight that low MUAC children have a higher risk of death.

Children with low MUAC benefit from treatment and when given an adequate therapeutic diet, show rapid catchup growth and rapidly increase their MUAC [25, 28, 29]. As a large, high-risk group that respond well to treatment, the detection, referral and treatment of these children should be regarded as a top public health priority population. That they are on average younger or more stunted, and for that reason have a higher risk of death even in absence of associated severe wasting must not be used as an argument to refuse to consider them as a high priority group. Arguably, young age and associated stunting may have additional effects on body composition, making these children especially vulnerable to wasting [30]. This high risk group is easy to identify which facilitates the detection and treatment of these high risk children and makes it easier to integrate within existing health programs, a key factor to achieve high treatment coverage. A pilot study has recently suggested that mothers can be involved in screening using MUAC, empowering them to actively seek treatment when needed [31]. This also has a promising potential to increase treatment coverage.

Instead of promoting the use of $\mathrm{WHZ}$ as an additional criterion to select children for treatment, greater sensitivity and specificity for mortality can be obtained by increasing the MUAC cut-off. This is logistically easier to implement and available data suggest it would be more effective to detect high risk children [13]. In this regard, the recently published results of a study from Sierra Leone, using a MUAC based protocol integrating treatment of moderate and severe malnutrition as a continuum and giving apparently excellent results [32] deserves special attention. This study included all children with a MUAC less than $125 \mathrm{~mm}$ in the program, presumably covering all children at risk of malnutrition related-death, as the curve describing the relationship between MUAC and mortality is flat above this cut-off [33]. Children with a MUAC between 115 and $125 \mathrm{~mm}$ received a less intensive treatment, which apparently was sufficient to prevent deaths during treatment. The future lies in a community-based screening by mothers of all children with a MUAC less than $125 \mathrm{~mm}$ coupled with a treatment wherein intensity depends on the MUAC level as was done in Sierra Leone [32].

\section{Conclusion}

While a better understanding of the mechanism behind the discrepancy between MUAC and WHZ is desirable, research in this area should not delay the implementation of programs aiming at effectively reducing malnutritionrelated deaths by prioritising the detection and treatment of children with low MUAC. 


\title{
Response to "Low mid-upper arm circumference identifies children with a high risk of death who should be the priority target for treatment"
}

Emmanuel Grellety ${ }^{1}$ and Michael H. Golden ${ }^{2}$

\author{
${ }^{1}$ Research Center Health Policy and Systems - International Health, School of Public Health, Université Libre de \\ Bruxelles, Bruxelles, Belgium \\ ${ }^{2}$ Department of Medicine and Therapeutics, University of Aberdeen, Aberdeen, Scotland
}

In the above Correspondence, Briend et al. have commented on our paper [1] which concludes that it is premature to abandon WHZ (weight-for-height Z-score) as a criterion for SAM (Severe Acute Malnutrition) particularly in countries where a large proportion of malnourished children fulfil the diagnostic criteria for SAM using the WHZ criterion but not with the MUAC (Mid-Upper Arm Circumference) criterion.

The main criticisms are:

1) MUAC is universally better at identifying children at risk of death than WHZ and is very good at identifying children with a high risk of death if left untreated.

2) Patient based studies are biased, truncated, not representative of the community and are therefore of little value.

3) Children who have a low WHZ are relatively healthy and have a low risk of death.

4) The failure to identify the same children by MUAC and WHZ simply reflects changes in body shape, in particular the relative length of the legs to the torso.

5) As children with a low WHZ are relatively healthy and are only identified as malnourished because they have longer legs they do not require treatment.

6) Children who have a low WHZ can easily be identified for treatment by increasing the cut-off point for MUAC.

7) WHZ is difficult to measure, wastes resources and consumes staff time inappropriately.

8) Our paper constitutes a threat to MUAC-only programs.

These same points have been repeatedly made elsewhere [34, 35] including the statement that using WHZ increases mortality and its use is unethical.

We reject each of these contentions and consider the views to be extreme, the logic flawed, the opinions based upon misinterpretation of data and the assertions misleading.

To be clear, the purpose of our paper is not to criticise the use or utility of MUAC. MHG has advocated for the use of MUAC as an independent criterion for the identification and admission of SAM children for more than 25 years and has consistently included MUAC criteria in all the protocols in which he has have been involved; we know of nobody who is "anti-MUAC", or is advocating that MUAC should not be used!

There is no doubt that the widespread use of MUAC has been a major advance in screening for malnourished children in the community, and should also be used by all clinicians and nurses in all health facilities if they have been properly trained and tested; this is what we teach and advocate. It is a simple method, uses cheap equipment that is easily portable, is rapid to make the measurement and very easily understood; however, its precision and accuracy are problematic [36-38]. There is no dispute about these advantages and attributes; our paper in no way challenges the use of MUAC.

We fully endorse the position taken by the senior advisors and heads of the head-quarters Nutrition Departments of Médecins Sans Frontières (MSF) [39] in their widespread use of MUAC only programs in acute emergency situations where very large numbers of malnourished children overwhelm national health services, and where an agency is focused particularly on malnutrition and has sufficient logistic, human and material resources to treat all the children so identified.

However, our advocacy for MUAC should not be equated with being anti-WHZ. Our paper does show that the discrepancy between WHZ and MUAC, that has been described from single or a few countries, is a universal phenomenon, that the direction of the discrepancy has been under-estimated in the past (WHO states that there is a $40 \%$ overlap [40]), that the discrepancy is greater for severe than moderate acute malnutrition and more importantly that the direction of the discrepancy varies markedly from country to country.

\section{Assertion 1. MUAC is universally better at identifying children at risk of death than WHZ and is very good at identifying children with a high risk of death if left untreated}

The principle argument that Briend et al. use to conclude that WHZ should be abandoned is that it generally has a lower area under a ROC curve than MUAC. ROC curve analysis is promoted in a way that trumps all other evidence which can then be dismissed. 
ROC analyses were originally used to determine appropriate cut-off criteria for different diagnostic tests to efficiently balance specificity and sensitivity for patients that had already been selected with a diagnostic goldstandard. In this case the anthropometric criteria are being used as diagnostic tests for future death. Pre-death is the condition being diagnosed, not malnutrition. The data for the analysis derives from cohorts of children followed up for many months and the areas under the curves for WHZ-scores and absolute MUAC examined to determine which better distinguishes those that subsequently survived and those that died. There are problems with this approach in terms of malnutrition and the interpretation of the data.

1) First, it is asserted that MUAC is a good predictor of future mortality. In fact, although the area under the MUAC-ROC curve is usually slightly greater than the WHZ-ROC curve, both are poor predictors of all-cause mortality with disappointingly low areas under their ROC curves; the differences are relatively small, often without a significant difference [41]. Those studying other diseases would dismiss both as being poor prognostic tools. This is in contrast to a ROC curve of already malnourished children which shows that including other measures of malnutrition such as serum albumin and transthyretin [23] converts a poor diagnostic tool to one with a ROC-curve comparable to tests used for other diseases.

2) A proportion of the children will have died from causes unrelated to nutrition; in other words these analyses examine the relative merits of MUAC and WHZ to predict all-cause mortality. We know that relatively few children die directly from severe malnutrition and underlying malnutrition contributes to between 25 and $50 \%$ of all childhood deaths [12]. Thus, more than half the deaths have no relationship with anthropometric malnutrition and could not be averted by nutritional intervention. To avert deaths caused by malnutrition we have no interest in using anthropometric tests to predict who will die from non-malnutrition related causes such as an accident. The large numbers of nonnutrition related deaths included in these analyses bias the ROC curves and complicate interpretation.

We want diagnostic tests that identify those who have malnutrition, are at increased risk of death because of the malnutrition and whose deaths can be averted by relatively short-term treatment. It is accepted that treatment that leads to weight and MUAC gain to normal levels will avert a proportion of the deaths. We do not know the proportion of malnutrition related deaths that could be averted and whether this is greater or less when WHZ or MUAC definitions are used.

3) Of necessity, in order to obtain sufficient incidents the follow up period has to be many months to several years. Death then occurs remotely in time from baseline measurement. The measurement is presumed to be predictive of a future status of the child rather than the current status and need for current nutritional treatment to avert death. Baseline or even "latest" anthropometric measures from a previous visit may quickly become outdated, leading to an unknown status at the time of death. This is a very serious limitation of such analyses particularly affecting an absolute measurement taken at baseline (such as MUAC) which is more likely to be below a cut off point at baseline and above the cut-off point by the time of death because most children grow with time. Measures of chronic malnutrition, poverty or deprivation are likely to become steadily more prognostic of future death than measures of baseline (or even latest measured) nutritional status as the length of follow up increases. This would favour measures such as height-for-age, weight-for-age and absolute MUAC than criteria that measure acute malnutrition and death shortly after the measurement. It is likely that improvement of current nutritional status will have a long lasting effect, for example see Isanaka et al. [42]. As low absolute MUAC is more likely in stunted than non-stunted children of the same age, the component of chronic malnutrition in absolute MUAC may boost its ROC curve for death much later during follow-up, but not necessarily the need for acute short term treatment. Different results are obtained if the latest anthropometric results are considered rather than baseline measurements [43].

4) As we have shown, MUAC and WHZ identify malnutrition in different children. By analogy, consider diagnostic tests for metabolic syndrome in adults. If we examined ROC curves for blood pressure, BMI, blood glucose and cholesterol against all-cause mortality they would all have different "areas under the curve" and perform relatively poorly because there will be deaths from carcinomas, infections, trauma etc. that have no relationship to metabolic syndrome. Such is the case with malnutrition. In this example those dying from carcinoma are likely to have a low BMI so that including cancer deaths in the analysis would reduce the ability of an increased BMI to predict death. Absolute-MUAC is lower in younger children because MUAC increases with age, but WHZ is much less affected by age. Suppose that malnutrition related 
deaths are the same with a low WHZ and with a low MUAC, but that older children are more likely to die from accidents such as drowning or falling from trees. As accidental death risk is highest in the older healthy active child, inclusion of these deaths will artificially suppress the WHZ-ROC curve by "diluting" the malnutrition related deaths with deaths of active normal children. Indeed, two to 3 year follow up of WHZ of older children may even show a protective effect of a low WHZ [41]! For example, in one study in Jamaica nutrition and psychosocial stimulation was associated with death in the intervention group due to accidents when the children became more active; there were no deaths in the non-intervened group (S. Grantham-McGregor, personal communication). ROC curves mislead when deaths are included in the analyses that are more common in well nourished older children.

The effects of confounding, age and length of follow up has been extensively investigated in Malawi [41]. Age and length of follow up affect the utility of different anthropometric parameters to predict death. Both MUAC and WHZ are better in children $<24$ months than in older children, and the various anthropometric markers have different capacities to predict long term (year 2 and 3) than shorter term death (first year). In most, but not all, of these analyses MUAC has a greater area under the ROC curve than other anthropometric indices. However, in this paper [41] ROC curve analyses do not distinguish statistically between WHZ and MUAC risks of death for many of the comparisons. It is incorrect to claim that MUAC-ROC is universally superior to WHZ-ROC, or that it is a good prognostic tool. Community studies are very difficult to undertake; the difficulties of interpretation are in addition to the problems of all long term studies of lost-to-follow-up subjects and other forms of bias.

5) The MUAC data is defined by a fixed cut-off point irrespective of the child's age or gender whereas WHZ is related to standard children of the same height and gender. MUAC is therefore strongly biased towards selecting shorter/younger children and biased against older children. As mortality from most causes (a notable exception is accidents) falls exponentially with age from 6 to 59 months in all societies a low MUAC is bound to identify more children that die than any standardised measure whether or not these are related to malnutrition. Indeed, it would be surprising if the ROC curves for absolute weight, or height were not better than WHZ; and even age itself is better than MUAC in some contexts [19]. This will be the case even if none of the deaths are related to nutritional status and nutritional therapy would not avert any deaths. Indeed, MUAC may predict deaths in highly developed societies because age is associated with a higher death rate in all societies. The argument that MUAC should be used exclusively to select children for therapeutic feeding because it is a "better" predictor of mortality than WHZ, could equally be used to select children for treatment of any condition more commonly causing death in younger children; for example, we could use MUAC data to give anti-malarial treatment if we did not have better methods of making the diagnosis of malaria.

Nevertheless, the bias in favour of younger children, because of their inherently increased risk of death, including death from malnutrition, is an undoubted advantage of using MUAC; this is not in contention. However, abandoning WHZ is at the expense of denying treatment to severely malnourished older/taller children whose lives and outlook for the future could be salvaged by therapeutic feeding.

6) As the prevalence of SAM and MAM increases in a population one would expect a higher proportion of deaths to be malnutrition related and fewer due to conditions unrelated to malnutrition. This should result in an increase in the area under the ROC curves for both MUAC and WHZ. This is not the case. Indeed, MUAC-ROC curves are even poor predictors of death in children admitted to hospital with severe malnutrition [23]. Patient curves are similar to those from community studies. One would expect that selection of children already malnourished would result in a ROC curve substantially better than those from the community.

Thus, although ROC-curves from community studies add to the evidence base their interpretation is complicated, open to bias, errors of timing and diagnosis and confounding. They should not be taken as definitive and used uncritically to advocate abandoning all children with a WHZ below -3 Z-scores, but with a normal MUAC, to their fate.

\section{Assertion 2: Patient based studies are biased, truncated, not representative of the community and are therefore of little value}

The second source of data on malnutrition related outcome comes from series of patients diagnosed as malnourished and followed during and after treatment. With all other diseases this is the way that diseasespecific mortality is determined in preference to using future all-cause mortality in the community. The other 
conditions have agreed case definitions to distinguish patients from non-patients, this is not the case with anthropometric malnutrition as there are two criteria currently in use and they identify different children.

As with community studies there are pitfalls with the use of patient data. The mortality will relate to the severity of the illness and to the quality of the treatment; the assumption needs to be made that those that die under treatment would be the most at risk if left untreated. This is a reasonable assumption, but is a potential problem when comparisons between different centres are made and nosocomial deaths cannot be identified; both the quality of care and severity of the condition are likely to vary across centres and regions.

However, the main problem with patient studies is the potential for ascertainment bias. For example where there is community screening with MUAC, but WHZ patients are admitted from hospital or health centre attendees, the WHZ patients are likely to be more severely affected or have already developed complications and therefore be at a higher risk of death when these groups are compared. Where both groups of patients are selected only from health centres or from the community, this type of bias is ameliorated.

The paper by Grellety et al. [21] is singled out for criticism on the basis that there is a "truncated" sample, an ascertainment bias and that it is not representative of the community. All studies use "truncated" samples, often children 6-59 months in this context and the results only apply to such a sample. Infants below 6 months and children above 59 months also become malnourished as well as adults, the elderly, prisoners etc. Truncation per se is not a valid criticism of a study. The results of this study apply to children admitted by the selection criteria used a priori at that time for admission; if different selection criteria are used, for example by extending admission to older, younger or shorter patients, of even omitting all children with SAM by WHZ, but not by MUAC, then different results may or may not have been obtained.

Briend et al. complain that a patient population does not represent the "community" - by which we presume is meant the total childhood population. This is also not a valid criticism. To address the problem of mortality due to malnutrition (and not other conditions) we do not want a representative sample of the "general population", which is mostly composed of healthy children without any anthropometric deficit and would not qualify for treatment anywhere. We do want to select the children who would benefit from treatment of malnutrition, and we are searching for ways to identify all such children. In other words all such studies examine a group of patients selected or excluded by predetermined criteria and their outcome observed; in this case children with either a low MUAC or a low WHZ were studied. These criteria are then the same as those applied to select those children in the community that require treatment; the "community" is not the target for specific interventions for SAM, although an often misunderstood "buzz word".

In all disease specific studies there is a potential problem with ascertainment bias. Briend et al. state that "children 6 months of age or older but less than $65 \mathrm{~cm}$ in length were only eligible for admission if their WHZ was less than -3Z". In fact the protocol followed in this study was the MSF protocol (internal document); this protocol repeatedly states that MUAC should only be used in children over $65 \mathrm{~cm}$. The MSF/ALIMA signatories to Briend et al. were the authors of this protocol and Briend, Manary and Guerrero the external advisors. It seems that the critics are criticising their own procedures! However, in this study, the reason for the very low admission of patients with a MUAC-only diagnosis was the nature of the population screened.

The speculation by Briend et al. concerning the numbers of children with a low MUAC (and a WHZ >-3Z) who were excluded from that study has not been correctly computed $[34,35]$. We have examined survey data of representative samples of 6-59 month old children randomly selected from the same area as the program (South Sudan). There were 140,249 children surveyed in the community; there were 8,449 children with SAM by WHZ, MUAC or both criteria. Of these children 4,668 had SAM by WHZ only. To examine the potential effect of the admission criteria we counted the number of children with MUAC $<115$, WHZ $>-3 Z$ who were less than $67 \mathrm{~cm}$. There were 358 such children in the whole community $(0.25 \%$ of the sample population). In other words of the 8,449 malnourished children in this community $4.2 \%$ would have been excluded if this admission criteria had been used. In contrast, if a MUAC only program had been implemented in this community, then 4,668 children with severe malnutrition would have been excluded from treatment. To exclude $55 \%$ of the malnourished children in this community would indeed have produced a "truncated" sample and been quite unrepresentative of SAM in the community. Attempts to discredit this paper for using a height cut-off depends upon a minor quibble compared to the consequences of denying 4,668 severely malnourished children from any treatment.

It may be thought that this is a particular situation because the prevalence of SAM by WHZ far exceeds that by MUAC in South Sudan. But this is precisely what our paper [1] illustrates would happen in countries such as the Philippians, Sri Lanka, Sudan and Senegal. Our data show that the problem with which Cambodia is struggling $[44,45]$ is more general than a local Cambodian or 
South Sudanese phenomenon. In our database of 1.4 million children [1] using a MUAC-only program would deny diagnosis or treatment to about $45 \%$ of all severely malnourished children. This in our view would be unethical if they could be efficiently indentified. However, as our paper demonstrates these problems are countryspecific. In some countries MUAC-only programs will identify most of the malnourished children and should be actively promoted, in others it will neglect most children in need of therapeutic feeding, abandon them to their fate and should not be instituted.

Use of patient data, such as that reported by Grellety et al. [21] and Isanaka et al. [20] provides important evidence for the selection of patients for treatment of malnutrition and for informing policy and should be honestly evaluated together with community data. Application of various admission criteria, is not a reason to dismiss the data as irrelevant; to do so arises from a concerted anti-WHZ agenda [34, 35] which attempts to discredit any evidence that questions the abandonment of WHZ as a valid criterion for treatment. Grellety et al's [21] results are legitimate and add to the evidence base in the same or better way than community studies. No matter what criticisms are levelled at the ascertainment of patients for this study the fact remains that one third of the children that died in this program would not have been admitted using MUAC only criteria. Such a result and other patient based studies cannot be simply dismissed by unfair criticism.

\section{Assertion 3: Children who have a low WHZ are relatively healthy and have a low risk of death}

There are no data to support this assertion. The first detailed study of malnutrition related death was in Latin America [46, 47]. Puffer and Serrano investigated over 35,000 childhood deaths and found that nearly 12,000 (34 \%) were due to malnutrition (Gomez classification or kwashiorkor). The risk of death fell dramatically from 3 months of age which would of course favour an absolute cut-off of any parameter which increases with age rather than one which is age/height standardised.

An exponential increase in the risk of death of children followed in the community with deterioration of both WHZ and MUAC was later shown by Pelletier [12]. However, it should be noted that SAM by weightfor-height in Pelletier's analysis is defined as $<80 \%$ (NCHS), which is not SAM but global acute malnutrition, compared with MUAC as $<110 \mathrm{~mm}$ so that the relative risks in this paper actually compare GAM with very severe acute malnutrition; the comparison is thus misleading. The data do not show that children with a low MUAC are at heighten risk whereas children with a low WHZ are "healthy".
A more recent analysis of 10 prospective community studies in Africa, Asia and South America of all-cause mortality showed that all degrees of underweight, stunting and wasting were associated with significantly higher death rates; a low WHZ was a much stronger determinant of mortality than either stunting or underweight. Children with SAM (WHZ <-3Z) were 11.6 times more likely to die than normally nourished children; for very severely wasted children ( $\mathrm{WHZ}<-4 \mathrm{Z}$ ) the hazard ratio for death was 22 times that of normal children [43]. These analyses were conducted using the $\mathrm{WHO}_{2006}$ growth standards, robust statistical methods and found that neither confounding nor cohort effects altered the results. The authors also conducted sensitivity analyses to determine the effect of carrying the last observed anthropometric measurement forward to the time of death (mean 8 weeks delay). MUAC was not reported in this study so no comparison is possible.

Even short term follow up of children in the community show excess mortality for those with a low WHZ/BMIZ; with a Z-score of $<-3$ about $5 \%$ of children are dead within 3 months, this increases to 15 to $20 \%$ at $<-4$ and $30 \%$ for those approaching -5Z [48]. Briend is a co-author so should not be unaware that his assertion that children with a low WHZ are "healthy" is misleading.

These data agree with clinical experience. The more wasted a patient by either criterion the more likely is death; observations that extend to adolescents (e.g. anorexia nervosa) and adults with cachexia or other measures of wasting. We do not comprehend how children with a WHZ lower than -3Z scores from the standards could be thought of as "healthy", let alone wealthy and undeserving of treatment.

\section{Assertion 4: The failure to identify the same children by MUAC and WHZ simply reflects changes in body shape, in particular the relative length of the legs to the torso}

Contrary to the assertion by Briend et al. we find that the each of the current explanations for the discrepancy, in particular the relative leg length focused upon by Briend, are all inadequate. Briend et al. misquote our paper and consistently misquote Roberfroid et al's paper $[34,35,49]$ which both show that long-legs have a minor effect on WHZ which is inadequate to explain the discrepancies between WHZ and MUAC in diagnosing SAM. There are many societies shown in our paper where long-legs cannot be the cause for the dramatic discrepancy between MUAC-SAM and WHZ-SAM. Attempts to understand the basis on body composition assessment in Ethiopia [50] have simply shown that WHZ is more closely related to body fat-free mass, and absolute-MUAC to fat mass when adjusted for height. MUAC is also related to height itself, so that the 
absolute MUAC is partly a composite measurement that is related to both wasting and stunting. Thirty to 50 days of outpatient therapeutic feeding has not yet been shown to correct stunting to our knowledge, although it may prevent deterioration [42].

The papers quoted by Briend et al. [7] showing that central fat is higher than peripheral fat in Puna, India further complicates the explanation as central fat should increase WHZ relative to MUAC and not the reverse as suggested by Briend et al. This is another misinterpretation.

\section{Assertion 5: As children with a low WHZ are relatively healthy and are only identified as malnourished because they have longer legs they do not require treatment}

Our conclusion that low WHZ and low absolute-MUAC have an additive effect is based on logic. That is the fact that the two criteria identify different children, not specifically on the literature per se as supposed in the criticism. Logically, if one measure was truly inferior and can therefore be dispensed with, the superior measure would identify most of those children already identified by the inferior measure; it would then be "superior" because it identifies additional children that die. This has not been shown to be the case. If different children's deaths contribute to the positive aspects of the MUACROC curve from the deaths contributing to the WHZROC curve then the two criteria are clearly additive. As the children with the deficits are in the main different it follows that the contribution to the positive aspects of the ROC curves are most likely to come from different deaths. Only if the same children's deaths contribute equally to both ROC curves with additional deaths augmenting the MUAC-ROC curves would Briend et al's argument have merit.

If a child dies with a low MUAC and a high WHZ the effect will be to improve the MUAC-ROC curve and weaken the WHZ-ROC curve. Alternatively if the child dies with a low WHZ and a MUAC above the cut-off point, then the effect will be to enhance the WHZ-ROC curve and depress the MUAC-ROC curve. It follows that the children that die will contribute differently to the two ROC curves if the dead children are themselves discordant for WHZ and MUAC. As the overlap between SAM by MUAC and WHZ is only about $16.5 \%$ it follows that the deaths attributed to one criterion are usually different deaths from the deaths attributed to the other criterion. It is likely that younger children dominate the MUAC-ROC curve and older children contribute more weight to the WHZ-ROC curve (diminished by accidental deaths etc.). Unless all the deaths are confined to children that satisfy both criteria the two criteria are additive and complementary and not proxies for the same risk of death. That being the case a MUAC-only program will neither detect children with a low WHZ nor prevent their deaths - they will become the "forgotten SAM children".

It should be noted that in the paper by Grellety et al. [21] the children with a low MUAC had a mean WHZ of -4.3 , whereas those with a MUAC above the cut-off had a WHZ of only $-3.8 Z$; the higher death rate in the MUAC group could equally well be ascribed to their lower WHZ as to their MUAC; they mostly had combined deficit which would account for the higher death rate (an additive effect).

\section{Assertion 6: Children who have a low WHZ can easily be identified for treatment by increasing the cut-off point for MUAC}

Briend et al. suggest that the solution to the excluded WHZ-children is simply increasing the MUAC cut-off point for admitting children to therapeutic feeding programs [13], and claim that this is logistically easier than using WHZ. The screening and "diagnosis" would indeed be simpler, but the implementation of treatment would be logistically very difficult if not impossible at scale with potentially negative effects. Increasing the cut-off point for MUAC in order to include the children with WHZ $<-3$ but a MUAC of over $115 \mathrm{~m}$ will result in admitting increasingly large numbers children who are not malnourished and are not thought to need acute treatment to avert death from malnutrition. There are relatively few children with SAM by either criterion; in our database only $3.6 \%$ of the population. Although, it could be argued that most diets in the poorer countries are lacking in several essential nutrients and that all would benefit from highly fortified food supplements, individual therapeutic feeding is not a feasible way to address this massive problem.

With a cut-off of $130 \mathrm{~mm} 17 \%$ of communitychildren would be admitted and if the cut-off is further increased to $135 \mathrm{~mm}$ nearly $30 \%$ of all the children in the community would be treated. With a cut off of $130 \mathrm{~mm}$ only $18 \%$ of those receiving treatment actually have SAM! It is clearly not "logistically simple" or feasible from a financial, material or human resource point of view to admit $30 \%$ of a community's children for therapeutic feeding. If there is an operational supplementary feeding program many of these additional children can be treated with a reduced or different ration. However, extending the cut-off beyond $125 \mathrm{~mm}$ results in an influx of large numbers of relatively healthy children for "treatment"; the increased work-load is to the detriment of all other essential health services and may thus increase mortality. Valid International has shown [51] that screening and referring children who are then rejected from a program, brings the whole program into disrepute with the community. Thus, 
although it is a superficially attractive to increase the MUAC cut-off to capture WHZ $<-3 Z$ children with a MUAC $>115$ into the program it is not realistic at scale.

Of more importance, analysis of our database shows that a MUAC cut-off point of $125 \mathrm{~mm}$ would still miss $24 \%$ of children with a WHZ $<-3$, and even a cut-off point of $130 \mathrm{~mm}$ would exclude $16 \%$ of severely malnourished children by WHZ. The assumption by Manary's group [32] that a MUAC cut of point of $125 \mathrm{~mm}$ will identify all the severely malnourished children with a $\mathrm{WHZ}<-3 \mathrm{Z}$ is incorrect.

Blanket feeding is warranted in relatively small areas at considerable cost by well resourced NGOs in the face of a famine situation [52], and even $25 \%$ of all children could possibly be enrolled in other relatively small areas by well-resourced, focused, emergency NGOs; but this would not be for a whole country using the existing health services. Blanket feeding or increasing the MUAC cut-off should not be advocated for those that are trying to scale up treatment for SAM; it is a priority to focus on averting death in those at highest risk which clearly includes those with a WHZ $<-3 Z$.

\section{Assertion 7: WHZ is difficult to measure, wastes resources and consumes staff time inappropriately}

We do agree that WHZ is not as easy to measure as MUAC and its use in community screening is impractical; hence our full endorsement of the approach used by MSF [39] in emergency situations by well resourced NGOs.

The extended and enthusiastic use of MUAC will continue; but this is not a reason to deny that there is a problem with the large residue of patients in the community with a low WHZ and a normal MUAC (45\% of all SAM cases). Our paper highlights the importance of finding simple ways of identifying and offering treatment to such children. When a problem is robustly and repeatedly denied through the use of misinformation and flawed arguments the chances of it being appropriately addressed are greatly diminished.

It is undoubted that MUAC screening and outpatient treatment have led to agencies achieving amazingly good results, relatively cheaply and at scale for up to $55 \%$ of severely malnourished children globally; the majority in some countries but the minority in others. However, those with SAM by WHZ that are not identified in the community can only be identified in facilities where weight and height can be measured at the present time.

The reported "coverage" of programs using MUAC screening omits all those additional severely malnourished children which would be identified were we to be able to identify those children with a low WHZ in the community. It is inescapable to conclude that the reported coverage rates miss up to $45 \%$ of malnourished children globally and give a false impression of the ability of these programs to address the totality of the problem of malnutrition in many communities.

It is sad that the authors advocate so strongly abandonment of WHZ, even in facilities with the capacity to assess WHZ. We consider this to be premature, pending advances in understanding and methods of identification of WHZ-only patients.

\section{Assertion 8: Our paper constitutes a threat to MUAC-only programs}

To consider our paper a threat to the continued and expanded use of MUAC is quite unjustified. We have strongly advocated for MUAC (and edema) data to be included in all nutrition surveys and that the total of malnutrition (MUAC and/or WHZ and/or edema) should be reported to show the real extent of the problem which is grossly under-estimated in current reports. We applaud the advances made in community screening and out-patient management.

Advocating retention of $\mathrm{WHZ}$ as an independent criterion as well as MUAC could only be construed as being against the use of MUAC by those that are determined to abandon WHZ as a diagnostic criterion for SAM. The two criteria are not in competition with each other. Because MUAC is easy to measure in the community it will continue to be widely used and it is unnecessary to justify this by claiming that children with a low WHZ are simply those with longer legs, are healthy, that children with a low WHZ are not also at risk of all the adverse outcomes of being severely malnourished or that older malnourished children are of no consequence.

In summary, MUAC is the only existing simple, practical measurement that is available for community screening of children for malnutrition. Its use for identifying children and offering them treatment has indeed been a major advance. We completely agree that the treatment of the severely malnourished is a major public health priority. We disagree that our paper represents a threat to these programs in any way. It does demand that we find an adequate explanation for the discrepancy. We maintain that it is premature to discard $\mathrm{WHZ}$ as a diagnostic criterion for severe malnutrition. To do so would result in very large numbers of children being ineligible for care without any evidence that this is a safe policy; given the exponential increase in mortality with decreasing WHZ it is clear that these children are at greatly heightened risk of death. Increasing the cut-off for MUAC is not a satisfactory solution. Nevertheless, there is clearly a problem in identifying those children with a low WHZ that are not identified with the MUAC criteria and methods need to be developed that identify these children. There are countries such as Cambodia that are already facing this problem and others that our data predicts will experience the same problem for which a satisfactory solution has not been forthcoming. 


\section{Abbreviations}

MUAC: Mid-upper arm circumference; SAM: Severe acute malnutrition; WHZ: Weight-for-height z-score

\section{Acknowledgements}

Not applicable.

\section{Funding}

Not applicable.

\section{Availability of data and materials}

Not applicable.

\section{Authors' contributions}

AB wrote the first draft of this comment. All other co-authors reviewed and edited successive drafts, when appropriate. All authors reviewed and approved the final version of the manuscript.

\section{Authors' information}

Not applicable.

\section{Competing interests}

The authors declare that they have no competing interests.

\section{Consent for publication}

Not applicable.

\section{Ethics approval and consent to participate}

Not applicable.

\section{Author details}

${ }^{1}$ Tampere Centre for Child Health Research, University of Tampere and Tampere University Hospital, Lääkärinkatu 1, 33014 Tampere, Finland. ${ }^{2}$ Department of Nutrition, Exercise and Sports, Faculty of Science, University of Copenhagen, Rolighedsvej 30, DK-1958 Frederiksberg, Denmark. ${ }^{3}$ Action Against Hunger UK, London SE10 8JA, UK. ${ }^{4}$ Médecins Sans Frontières, CH-1211 Genève 2, Switzerland. ${ }^{5}$ Valid International, 35 Leopold Street, Oxford OX4 1TW, UK. ${ }^{6}$ Center for Epidemiology, Biostatistics and Clinical Research, School of Public Health, Université Libre de Bruxelles, Brussels, Belgium. ' International Rescue Committee, 122 E. 42nd St, New York, NY 10168, USA. ${ }^{8}$ KEMRI/Wellcome Trust Research Program, Kilifi 80108, Kenya. ${ }^{9}$ Valid International, 35 Leopold Street, Oxford OX4 1TW, UK. ${ }^{10}$ Alliance for International Medical Action, Dakar, Senegal. ${ }^{11}$ Tampere Centre for Child Health Research, University of Tampere, Tampere, Finland. ${ }^{12}$ Institut de Recherche Santé et Société, Université Catholique de Louvain, 1200 Brussels, Belgium. ${ }^{13}$ Médecins Sans Frontières, Brussels, Belgium. ${ }^{14}$ Contrevoz 01300, France. ${ }^{15}$ Action Against Hunger US, New York, NY 10004, USA. ${ }^{16}$ Action Against Hunger UK, London SE10 8JA, UK. ${ }^{17}$ Médecins Sans Frontières, Paris, France. ${ }^{18}$ London School of Hygiene and Tropical Medicine, London, UK. ${ }^{19}$ Department of Pediatrics, Washington University in St. Louis, St. Louis, MO, USA. ${ }^{20}$ School of Public Health and Family Medicine, University of Malawi College of Medicine, Blantyre, Malawi. ${ }^{21}$ KEMRI/Wellcome Trust Research Program, Kilifi 80108, Kenya. ${ }^{22}$ Brixton Health, Llawryglyn, Powys SY17 5RJ, UK. ${ }^{23}$ Alliance for International Medical Action, Dakar, Senegal. ${ }^{24}$ Action Against Hunger US, New York, NY 10004, USA. ${ }^{25}$ Médecins Sans Frontières, Barcelona, España. ${ }^{26}$ Alliance for International Medical Action, Dakar, Senegal. ${ }^{27}$ Médecins Sans Frontières, Amsterdam, Netherlands. ${ }^{28}$ Accion Contra el Hambre, 28002 Madrid, Spain. ${ }^{29}$ DG ECHO, Bureau régional Afrique de I'Ouest, Dakar, Sénégal.

\section{Received: 25 March 2016 Accepted: 7 October 2016}

\section{Published online: 21 October 2016}

\section{References}

1. Grellety E, Golden MH. Weight-for-height and mid-upper-arm circumference should be used independently to diagnose acute malnutrition: policy implications. BMC Nutr. 2016;2:10.

2. Meyer R, Marino L. Identifying acute malnutrition - do we have an answer for policy makers? BMC Nutr. 2016;2:20.

3. Bern C, Nathanail L. Is mid-upper-arm circumference a useful tool for screening in emergency settings? Lancet. 1995;345(8950):631-3.
4. Lindtjørn B. Measuring acute malnutrition: a need to redefine cut-off points for arm circumference? Lancet. 1985;2(8466):1229-30.

5. Carter EP. Comparison of weight:height ratio and arm circumference in assessment of acute malnutrition. Arch Dis Child. 1987;62(8):833-5.

6. Post CL, Victora CG. The low prevalence of weight-for-height deficits in Brazilian children is related to body proportions. J Nutr. 2001;131(4):1290-6.

7. Lakshmi S, Metcalf B, Joglekar C, Yajnik CS, Fall CH, Wilkin TJ. Differences in body composition and metabolic status between white U.K. and Asian Indian children (EarlyBird 24 and the Pune Maternal Nutrition Study). Pediatr Obes. 2012;7(5):347-54.

8. D'Angelo S, Yajnik CS, Kumaran K, Joglekar C, Lubree H, Crozier SR, et al. Body size and body composition: a comparison of children in India and the UK through infancy and early childhood. J Epidemiol Community Health. 2015;69(12):1147-53.

9. Bogin B, Varela-Silva MI. Leg length, body proportion, and health: a review with a note on beauty. Int J Environ Res Public Health. 2010;7(3):1047-75.

10. Wells JC, Cortina-Borja M. Different associations of subscapular and triceps skinfold thicknesses with pathogen load: an ecogeographical analysis. Am J Hum Biol. 2013;25(5):594-605.

11. Bartz S, Mody A, Hornik C, Bain J, Muehlbauer M, Kiyimba T, et al. Severe acute malnutrition in childhood: hormonal and metabolic status at presentation, response to treatment, and predictors of mortality. J Clin Endocrinol Metab. 2014;99(6):2128-37.

12. Pelletier DL. The relationship between child anthropometry and mortality in developing countries: implications for policy, programs and future research. J Nutr. 1994;124(10 Suppl):2047S-81S.

13. Briend A, Maire B, Fontaine O, Garenne M. Mid-upper arm circumference and weight-for-height to identify high-risk malnourished under-five children. Matern Child Nutr. 2012;8(1):130-3.

14. Schwinger C, Fadnes LT, Van den Broeck J. Using growth velocity to predict child mortality. Am J Clin Nutr. 2016;103(3):801-7.

15. Mwangome MK, Fegan G, Fulford T, Prentice AM, Berkley JA. Mid-upper arm circumference at age of routine infant vaccination to identify infants at elevated risk of death: a retrospective cohort study in the Gambia. Bull WHO. 2012;90(12):887-94.

16. Bairagi R. On validity of some anthropometric indicators as predictors of mortality. Am J Clin Nutr. 1981;34(11):2592-4.

17. Alam N, Wojtyniak B, Rahaman MM. Anthropometric indicators and risk of death. Am J Clin Nutr. 1989;49(5):884-8.

18. Vella V, Tomkins A, Ndiku J, Marshal T, Cortinovis I. Anthropometry as a predictor for mortality among Ugandan children, allowing for socio-economic variables. Eur J Clin Nutr. 1994;48(3):189-97.

19. Van den Broeck J, Eeckels R, Massa G. Validity of single-weight measurements to predict current malnutrition and mortality in children. J Nutr. 1996;126(1):113-20.

20. Isanaka S, Guesdon B, Labar AS, Hanson K, Langendorf C, Grais RF. Comparison of clinical characteristics and treatment outcomes of children selected for treatment of severe acute malnutrition using mid upper arm circumference and/or Weight-for-Height Z-Score. PLoS One. 2015;10(9):e0137606.

21. Grellety E, Krause LK, Shams Eldin M, Porten K, Isanaka S. Comparison of weight-for-height and mid-upper arm circumference (MUAC) in a therapeutic feeding programme in South Sudan: is MUAC alone a sufficient criterion for admission of children at high risk of mortality? Public Health Nutr. 2015;18(14):2575-81.

22. Briend A, Dykewicz C, Graven K, Mazumder RN, Wojtyniak B, Bennish M. Usefulness of nutritional indices and classifications in predicting death of malnourished children. Br Med J. 1986;293(6543):373-5.

23. Dramaix M, Hennart P, Brasseur D, Bahwere P, Mudjene O, Tonglet R, et al. Serum albumin concentration, arm circumference, and oedema and subsequent risk of dying in children in central Africa. Br Med J. 1993;307(6906):710-3.

24. Berkley J, Mwangi I, Griffiths K, Ahmed I, Mithwani S, English M, et al. Assessment of severe malnutrition among hospitalized children in rural Kenya: comparison of weight for height and mid upper arm circumference. JAMA. 2005;294(5):591-7.

25. Dale NM, Myatt M, Prudhon C, Briend A. Using mid-upper arm circumference to end treatment of severe acute malnutrition leads to higher weight gains in the most malnourished children. PLoS One. 2013;8(2):e55404.

26. Briend A, Zimicki S. Validation of arm circumference as an indicator of risk of death in one to four year old children. Nutr Res. 1986;6(3):249-61. 
27. Briend A, Garenne M, Maire B, Fontaine O, Dieng K. Nutritional status, age and survival: the muscle mass hypothesis. Eur J Clin Nutr. 1989;43(10):715-26.

28. Goossens S, Bekele Y, Yun O, Harczi G, Ouannes M, Shepherd S. Mid-upper arm circumference based nutrition programming: evidence for a new approach in regions with high burden of acute malnutrition. PLoS One. 2012;7(11):e49320.

29. Fabiansen C, Phelan KP, Cichon B, Ritz C, Briend A, Michaelsen KF, et al. Short children with a low midupper arm circumference respond to food supplementation: an observational study from Burkina Faso. Am J Clin Nutr. 2016;103(2):415-21.

30. Briend A, Khara T, Dolan C. Wasting and stunting-similarities and differences: policy and programmatic implications. Food Nutr Bull. 2015;36(1 Suppl):S15-23.

31. Blackwell N, Myatt M, Allafort-Duverger T, Balogoun A, Ibrahim A, Briend A. Mothers Understand And Can do it (MUAC): a comparison of mothers and community health workers determining mid-upper arm circumference in 103 children aged from 6 months to 5 years. Arch Public Health. 2015;73(1):26.

32. Maust A, Koroma AS, Abla C, Molokwu N, Ryan KN, Singh L, et al. Severe and moderate acute malnutrition can be successfully managed with an integrated protocol in Sierra Leone. J Nutr. 2015;145(11):2604-9.

33. Myatt M, Khara T, Collins S. A review of methods to detect cases of severely malnourished children in the community for their admission into communitybased therapeutic care programs. Food Nutr Bull. 2006;27(3 Suppl):S7-23.

34. EN-Net. WFH versus MUAC. 2015. Emergency Nutrition Network. http://www. en-net.org/question/1915.aspx.

35. EN-Net. Only MUAC for admission and discharge? 2015. Emergency Nutrition Network. http://www.en-net.org/question/1922.aspx

36. Ayele B, Aemere A, Gebre T, Tadesse Z, Stoller NE, See CW, et al. Reliability of measurements performed by community-drawn anthropometrists from rural Ethiopia. PLoS One. 2012;7:e30345.

37. Johnson R. MUAC measurement - Innovative new tools needed. 2016. 26-72016. https://nutritionrapidsms.wordpress.com/2016/01/29/muacmeasurement-innovative-new-tools-needed/.

38. Saeed HA, Mogendi JB, Akparibo R, Kolsteren P. Reliability of mid-upper arm circumference measurements taken by community health nurses. Curr Res Nutr Food Sci Jour. 2015;3:26-35.

39. Phelan $P Q$, Lanusse C, van der Kam S, Delchevalerie P, Avril N, Hanson K. Simplifying the response to childhood malnutrition: MSF's experience with MUAC-based (and oedema) programming. Field Exchange. 2015;50:108-16.

40. WHO. WHO child growth standards and the identification of severe acute malnutrition in infants and children A Joint Statement by the World Health Organization and the United Nations Children's Fund. 2009. WHO. http://www. who.int/nutrition/publications/severemalnutrition/9789241598163_eng.pdf.

41. Pelletier DL, Low JW, Johnson FC, Msukwa LA. Child anthropometry and mortality in Malawi: testing for effect modification by age and length of follow-up and confounding by socioeconomic factors. J Nutr. 1994;124:2082S-105S.

42. Isanaka S, Nombela N, Djibo A, Poupard M, Van BD, Gaboulaud V, et al. Effect of preventive supplementation with ready-to-use therapeutic food on the nutritional status, mortality, and morbidity of children aged 6 to 60 months in Niger: a cluster randomized trial. JAMA. 2009;301:277-85.

43. Olofin I, McDonald CM, Ezzati M, Flaxman S, Black RE, Fawzi WW, et al. Associations of suboptimal growth with all-cause and cause-specific mortality in children under five years: a pooled analysis of ten prospective studies. PLoS One. 2013;8:e64636.

44. Fiorentino M, Sophonneary P, Laillou A, Whitney S, de Groot R, Perignon M, et al. Current MUAC cut-offs to screen for acute malnutrition need to be adapted to gender and age: the example of Cambodia. PLoS One. 2016;11:e0146442.

45. Laillou A, Prak S, de Groot R, Whitney S, Conkle J, Horton L, et al. Optimal screening of children with acute malnutrition requires a change in current WHO guidelines as MUAC and WHZ identify different patient groups. PLOS One. 2014;9:e101159.

46. Puffer RR, Serrano CV. Patterns of mortality in childhood: report of the InterAmerican Investigation of Mortality in Childhood, Paho Scientific Publication no. 262, 1-470. Washington: Pan American Health Organization; 1973. http:// www.popline.org/node/518356.

47. Puffer RR, Serano CV. The role of nutritional deficiency in mortality : findings of the Inter-American investigation of mortality in childhood. Bol Ofic Sanit Panam. 1973;7:1-25.

48. O'Neill SM, Fitzgerald A, Briend A, Van Den Broeck J. Child mortality as predicted by nutritional status and recent weight velocity in children under two in rural Africa. J Nutr. 2012;142:520-5. doi:10.3945/jn.111.151878.

49. Roberfroid D, Huybregts L, Lachat C, Vrijens F, Kolsteren P, Guesdon B. Inconsistent diagnosis of acute malnutrition by weight-for-height and mid-upper arm circumference: contributors in 16 cross-sectional surveys from South Sudan, the Philippines, Chad, and Bangladesh. Nutr J. 2015;14:86.

50. Grijalva-Eternod CS, Wells JC, Girma T, Kaestel P, Admassu B, Friis H, et al. Midupper arm circumference and weight-for-length z scores have different associations with body composition: evidence from a cohort of Ethiopian infants. Am J Clin Nutr. 2015;102:593-9.

51. Guerrero S, Myatt M, Collins S. Determinants of coverage in communitybased therapeutic care programmes: towards a joint quantitative and qualitative analysis. Disasters. 2010;34:571-85.

52. Defourny I, Minetti A, Harczi G, Doyon S, Shepherd S, Tectonidis M, et al. A large-scale distribution of milk-based fortified spreads: evidence for a new approach in regions with high burden of acute malnutrition. PLoS One. 2009;:4:e5455.

\section{Submit your next manuscript to BioMed Central and we will help you at every step:}

- We accept pre-submission inquiries

- Our selector tool helps you to find the most relevant journal

- We provide round the clock customer support

- Convenient online submission

- Thorough peer review

- Inclusion in PubMed and all major indexing services

- Maximum visibility for your research

Submit your manuscript at www.biomedcentral.com/submit
C Biomed Central 